\title{
¿Barreras invisibles? Actitudes de los estudiantes universitarios ante sus compañeros con discapacidad
}

\section{Invisible barriers? College student's attitudes towards peers with disabilities}

\author{
Eugenia GONZÁLEZ CORTÉS y Sergio ROSES CAMPOS \\ Universidad de Málaga
}

Recibido: Junio 2014

Evaluado: Julio 2014

Aceptado: Julio 2014

\section{Resumen}

El presente trabajo analiza los conocimientos, opiniones y actitudes de los estudiantes universitarios sobre sus compañeros con discapacidad. La integración educativa y social de estos estudiantes es objeto de preocupación y estudio en todo el sistema educativo español, incluida la enseñanza superior en la que 21.942 universitarios con discapacidad se encontraban matriculados en el curso 2013-2014. El estudio se realiza en el marco de dos Proyectos de Innovación Educativa $^{1}$ de la Universidad de Málaga en los que se examinaron las actitudes del personal docente e investigador, el alumnado en general y el alumnado con discapacidad de esta institución. En este artículo se recogen las perspectivas de los estudiantes sin discapacidad, necesarias para medir el grado de aceptación dentro de las aulas universitarias. Se empleó una encuesta suministrada mediante correo electrónico a la totalidad de la población de estudiantes de la Universidad de Málaga $(\mathrm{N}=32.464)$. El tamaño de la muestra fue de 620 estudiantes, por lo que trabajamos con un margen de error del 3,9\% para la estimación de los porcentajes con un nivel de confianza del 95\%. Los datos, analizados mediante análisis estadístico, visibilizan la importancia de un elevado grado de información y conocimiento así como de una experiencia directa con la discapacidad. Ambas circunstancias se traducen en actitudes más positivas y favorables. Tan solo el $20 \%$ de los alumnos manifestó haber recibido en alguna ocasión cualquier tipo de información sobre este asunto por parte de la Universidad; no obstante, la mayoría valora positivamente la presencia de alumnos con discapacidad en la Universidad, la posibilidad de contar ellos como compañeros de clase y el enriquecimiento que supone esta convivencia dentro del aula. Se discuten posibles acciones para mejorar las actitudes hacia los estudiantes con discapacidad. Además, se plantea incluir una perspectiva que promueva el empoderamiento de estos estudiantes.

Palabras clave: discapacidad, universidad; integración; inclusión educativa; actitudes.

\footnotetext{
${ }^{1}$ PIE 10-135 y PIE 13-103.
} 


\begin{abstract}
This paper analyses university students' knowledge, opinions and attitudes towards peers with disabilities. The integration of students with disabilities in Education is an object of concern and research in the whole Spanish Education System, including Higher Education where 21,942 people with disabilities were students in the academic course 2013-2014. This research belongs to two projects on Innovation in Education for the University of Málaga. Attitudes of professors, students without disabilities and students with disabilities were examined. This paper shows the viewpoint of students without disabilities, which is necessary in order to observe the acceptance inside the classes. An email-survey was conducted in the whole University of Malaga's student population $(\mathrm{N}=32.464)$. The sample size was 620 , so we had a confidence interval of $3.9 \%$ with a confidence level of $95 \%$. Statistical analysis was conducted. Data showed the relevance of higher levels of information and direct experience with the disability: those two variables correlated to more positive attitudes toward the students with disabilities. Only the $20 \%$ of the students said they had received any kind of information about disability from the university sometime, however most of the sample was favorable to the presence of students with disabilities in the university, to the possibility of sharing classwork with them and to the enrichment that coexistence inside the class provides. New actions in order to enhance student's attitudes toward students with disabilities were discussed, so the paper suggests including a new point of view that promotes the empowerment of those students.
\end{abstract}

Keywords: disability, university, integration, inclusive education, attitudes.

Se han identificado significativas barreras que dificultan a los estudiantes con discapacidad la participación, el progreso y el éxito en la Universidad (Moswela y Mukhopadhyay, 2011). Sin embargo, la literatura académica en el ámbito de la discapacidad y la enseñanza es cada día más numerosa, como lo son los intentos por normalizar la integración de todos los estudiantes en el aula y la evolución del marco legislativo en este mismo sentido. En la educación universitaria, la LOU, y, sobre todo la $\mathrm{LOMLOU}^{2}$, que la modifica, han contemplado también los derechos de las personas con discapacidad en su articulado (Disposición Adicional vigésimo cuarta "De la integración de estudiantes con discapacidad en las Universidades").

Más allá de los mandatos legales genéricos, y con el fin de mejorar la respuesta educativa que se proporciona a los estudiantes con discapacidad, se han publicado documentos dirigidos a profesores y alumnos en muchas instituciones universitarias. Así, por ejemplo, pueden citarse la Guía de adaptaciones y recursos para estudiantes con discapacidad, de la UNED (Andreu, Pereira y Rodríguez, 2010); las Pautas básicas para facilitar la integración de las personas con discapacidad, de la Universidad Complutense de Madrid (Martínez Liébana et ál., 2004) o la propia Guía de orientación al profesorado del alumnado con discapacidad en la Universidad de Málaga (Luque y Rodríguez, 2007). La mayoría de universidades españolas cuentan con servicios y unidades de atención a este alumnado específico, que han hecho que

\footnotetext{
${ }^{2}$ Ley Orgánica 4/2007, de 12 de abril, por la que se modifica la Ley Orgánica 6/2001, de 21 de diciembre, de Universidades.
} 
proliferen los manuales en esta línea. No sólo se ha considerado a los estudiantes, sino a todos los demás miembros de la comunidad universitaria; así, en enero de 2014 el CERMI (Comité Español de Representantes de Personas con Discapacidad) elaboraba un modelo de reglamento de medidas de acción positiva para el acceso y apoyo al personal docente e investigador con discapacidad, que fue remitido a todas las universidades españolas solicitando su aprobación como normativa propia (CERMI, 2014).

Si bien estas actuaciones son importantes, otro de los aspectos cruciales para la integración e inclusión social de los alumnos con discapacidad son las relaciones interpersonales que establecen con el resto de sus compañeros (Bueno y Mirambel, 2003; Polo y López, 2006; Alonso, Navarro y Lirón, 2008; Santos y Lorenzo, 2010). Estudios recientes han indicado que los jóvenes universitarios muestran una actitud positiva hacia sus compañeros con discapacidad (Suriá, 2011; Suriá, Bueno y Rosser, 2011), si bien es preciso conocer las razones determinantes de las conductas inclusivas. Novo, Muñoz y Calvo (2011) tratan por ello de identificar cuáles son estas razones, en un estudio donde concluyen que los valores sociales presentes mayoritariamente en Occidente constituyen la variable más influyente en la intención de ayudar a las personas con discapacidad, mientras que las actitudes personales, aunque predisponen de manera favorable, no muestran una influencia significativa. Los resultados de esta investigación muestran, asimismo, que los estudiantes prefieren que la labor de ayuda recaiga sobre las instituciones. Novo y Muñoz (2012) en un trabajo más reciente, realizado en la Universidad de A Coruña, alcanzan resultados esperanzadores que muestran la necesidad de que las instituciones públicas promuevan políticas educativas inclusivas en las Universidades. Los autores confirman, de nuevo, que la convivencia es determinante para mejorar las actitudes hacia la discapacidad y que de ella depende la existencia de un mayor grado de sensibilización. En 2013, Novo y López utilizan como marco de referencia la Teoría del Comportamiento Planeado (Ajzen, 1991), aplicada al estudio de las actitudes hacia la discapacidad, según la cual existen tres factores determinantes en la predisposición a prestar colaboración y ayuda a las personas con discapacidad: 1) la actitud hacia la inclusión o la predisposición del individuo hacia la discapacidad; 2) las normas sociales o las normas subjetivas y 3) el control percibido. En 2014, Luque de la Rosa y Gutiérrez evalúan en el contexto universitario italiano la percepción de los estudiantes sobre la integración del alumnado con discapacidad, mediante un estudio efectuado en la Universidad de Bolonia. Una vez más, se hallan unas ideas y actitudes favorables hacia la integración educativa y social, aunque insisten en que esta tarea se muestra aún inconclusa.

Se hace necesario ir más allá en esta línea de investigación para ofrecer la percepción de los tres sectores principales que conforman toda comunidad universitaria, interpelando además de al alumnado en general, a los estudiantes con discapacidad, al personal docente e investigador y al personal de administración y servicios. Sánchez Palomino $(2009,2011)$ recoge todos estos puntos de vista en un análisis comparativo realizado en la Universidad de Almería. En su investigación de nuevo se observa que los distintos colectivos muestran una actitud facilitadora de la integración educativa y social. 
No hemos hallado en el contexto español estudios que describan las características de un colectivo que, a juzgar por las tasas de matriculación, crece año tras año en todas las universidades españolas. En 2013/14 un total de 21.942 universitarios con discapacidad se matricularon en alguna de las 76 universidades españolas; una cifra que supone un incremento del $9 \%$ respecto al curso académico anterior, en el que se encontraban matriculados 19.953. El estudio se hace aún más pertinente en Andalucía, que registra una cifra de 2.277, con las Universidades de Sevilla (615) y Málaga (517) a la cabeza. Sólo superadas en todo el territorio nacional por las Universidades de Valencia y Murcia (con 1.117 y 911 alumnos con discapacidad matriculados, respectivamente), la Universitat Oberta de Catalunya (793), la Complutense de Madrid (738) y la de Barcelona (654). Más de 9.160 cursan alguna titulación en la UNED (Fundación Universia, 2014).

En la literatura anglosajona, destacan interesantes aportaciones sobre las relaciones interpersonales y las actitudes hacia la discapacidad, tanto de estudiantes como de docentes; durante la década de los ochenta, enfocan su atención en el área de la educación primaria y secundaria (Frith y Mitchell, 1981; Center y Ward, 1987), aunque sorprenden, por su anticipación en el tiempo, las investigaciones de Clore y Jeffery (1972), Evans (1976) y Stovall y Sedlacek (1983). Estos últimos encontraron claras diferencias de actitud en función de la situación y de la discapacidad física observada; se midieron las reacciones hacia los estudiantes ciegos y aquellos otros que iban en silla de ruedas (en situaciones académicas, las actitudes eran peores hacia las personas con discapacidad visual mientras que las reacciones hacia los segundos fueron más desfavorables en situaciones sociales). Existen otras variables que influyen en las actitudes. Diversos estudios indican que la Universidad, en ocasiones, no está suficientemente preparada para incluir al alumnado con discapacidad; así, por ejemplo, Gitlow (2001) refleja la importancia del asesoramiento y formación de los docentes para que sean estos quienes mejoren la respuesta hacia la diversidad. Se hace necesario el conocimiento por parte del profesorado de metodologías pedagógicas que favorezcan la inclusión, el conocimiento de la discapacidad propiamente dicha y, en el marco institucional, la existencia de infraestructuras para atender la diversidad. Por todas estas razones, algunos autores (Borland y James, 1999; Reindal, 1995, y Tinklin y Hall, 1999) consideran que a pesar de que existan normativas que regulan el derecho de este alumnado para recibir una educación de calidad y en igualdad de oportunidades, el ambiente universitario puede ser en algunos casos discapacitador (en Moriña Diez et. al, 2013).

En cualquier caso, desde muy temprano se observa que ni la información disponible sobre la discapacidad ni el contacto con personas discapacitadas son, por sí solas, condiciones suficientes para provocar cambios de actitud entre la población, sino que ambas circunstancias deben combinarse (Anthony, 1972). En 1986 Yuker y Block recopilan en una breve monografía toda la investigación que, en el transcurso de más de dos décadas y desde 1960 hasta 1985, habían medido las actitudes hacia las personas con discapacidad. Sirva como indicador de que el interés en esta temática goza de más antigüedad que la observada en el contexto español. 
Tal y como se ha hecho patente en la revisión de la literatura científica, los trabajos disponibles han estudiado el fenómeno a través de las poblaciones de universidades específicas. Con la finalidad de seguir aportando evidencias empíricas que apoyen y provean de validez a los resultados hallados en distintos lugares y momentos - a falta de que surjan investigaciones con recursos para abordar al conjunto del sistema español-, es necesario continuar realizando análisis en diferentes comunidades universitarias. Atendiendo a esta motivación, nuestro estudio plantea los siguientes objetivos a partir del caso de la Universidad de Málaga.

- Examinar las actitudes de los estudiantes hacia la inclusión de los alumnos con discapacidad en la universidad.

- Observar, por su especial importancia en el aprendizaje, las actitudes de los estudiantes ante la posibilidad de trabajar colaborativamente con compañeros que presentan algún tipo de discapacidad, así como su percepción sobre el rendimiento académico de este colectivo.

- Determinar si el conocimiento sobre la discapacidad (experiencia directa y grado de información) por parte de los estudiantes puede favorecer el desarrollo de actitudes más favorables hacia sus compañeros con discapacidad.

De acuerdo con los dos primeros objetivos del estudio, planteamos tres preguntas de investigación:

- RQ1: ¿Qué opinan los estudiantes sobre la posibilidad de trabajar en un grupo con estudiantes con discapacidad y sobre el rendimiento académico de estos compañeros?

- RQ2: ¿Cuál y cómo es la experiencia de los estudiantes en cuanto a relaciones en el aula con compañeros con discapacidad?

- RQ3: ¿Qué grado de información sobre la discapacidad presentan los estudiantes?

Asimismo, basándonos en los hallazgos reseñados en la literatura, y de acuerdo con el primer y tercer objetivo de la investigación, podemos enunciar tres hipótesis:

- H1: Los estudiantes presentan actitudes favorables hacia la inclusión de personas con discapacidad en la universidad.

- H2: Las actitudes favorables hacia el colectivo de estudiantes con discapacidad estarán asociadas positivamente con el grado de información recibido sobre discapacidad.

- H3: Los estudiantes que tienen un conocido con discapacidad en su entorno cercano presentarán actitudes más favorables hacia el trabajo en grupo y el rendimiento académico de alumnos con discapacidad.

\section{Metodología}

La investigación se erigió a partir de una metodología cuantitativa, concretamente se realizó una encuesta online que se suministró a la población del estudio, constituida 
por los estudiantes de la Universidad de Málaga $(\mathrm{N}=32.464)^{3}$. Debido al gran número de investigaciones realizadas en la universidad, el colectivo estudiantil es de difícil acceso cuando se procede mediante comunicaciones electrónicas, por ello, con la finalidad de obtener el mayor número posible de respuestas, se envió la encuesta a la totalidad de la población a través de una lista de distribución oficial de la universidad. El número de alumnos de la muestra ascendió a 620. Por tanto, trabajamos con un margen de error del 3,9\% para la estimación de los porcentajes con un nivel de confianza del 95\%. Las características de la muestra del estudio son las siguientes:

El 51,8\% de los encuestados era mujer. El 46,7\% estaba cursando uno de los dos primeros cursos de un grado; mientras que el $36,6 \%$ se encontraba en alguno de los cursos finales y el $6,8 \%$ en un tercer ciclo. De acuerdo al número de estudiantes por cada rama de conocimiento, la composición de la muestra de nuestro estudio (tabla 1) resultó bastante fiel a la estructura real de la población (cfr. Servicio Central de Informática, 2000) con la salvedad de que las ramas Técnica y Ciencias Experimentales quedaron algo infrarrepresentadas, mientras que el área de Ciencias de la Salud resultó bastante sobrerrepresentada en la muestra. Ninguno de los participantes presentaba alguna discapacidad ya que se suministró una encuesta específica en el marco del Proyecto de Innovación Educativa (PIE 10-135) a dicho colectivo con otros objetivos. No obstante, el 49,8\% de los estudiantes indicó que conoce a una persona con discapacidad que pertenece a su entorno más cercano. Se preguntó exclusivamente a este grupo qué tipo de relación les une: casi la mitad tiene un familiar con discapacidad; igual porcentaje mantiene una relación de amistad; el $26,2 \%$ tiene a un compañero de estudios; el $6,8 \%$, a uno de trabajo; apenas el $1 \%$, una relación sentimental, y el 14,6\% mantiene algún otro tipo de relación.

\begin{tabular}{lc}
\hline \multicolumn{1}{c}{ Rama de conocimiento } & Porcentaje \\
\hline Ciencias Sociales y Jurídicas & $57,5 \%$ \\
\hline Técnicas & $17,6 \%$ \\
\hline Ciencias de la Salud & $13,1 \%$ \\
\hline Humanidades & $8,2 \%$ \\
\hline Ciencias Experimentales & $3,6 \%$ \\
\hline
\end{tabular}

Tabla 1. Distribución de los estudiantes de la muestra por rama de conocimiento

Como ya se ha dicho previamente, empleamos una encuesta online, suministrada mediante correo electrónico a toda la población del estudio mediante una lista de distribución oficial de la universidad. El cuestionario incluyó preguntas cerradas sobre actitudes hacia los estudiantes con discapacidad y grado de información sobre discapacidad. Se usaron escalas tipo Likert de cinco puntos, preguntas con respuesta múltiple dicotómicas y preguntas con respuesta de tipo categórico. Antes de suministrar la encuesta, se realizó una prueba piloto entre un grupo de colaboradores

\footnotetext{
${ }^{3}$ Datos oficiales del último censo disponible.
} 
del proyecto. El enunciado de algunas preguntas fueron modificados para la ulterior realización de la encuesta.

El trabajo de campo definitivo se realizó durante el curso académico 2012/2013. Se usó la versión 20 del programa SPSS para el análisis de datos. Se utilizaron técnicas de estadística descriptiva para resolver las preguntas de investigación y correlaciones de Pearson y tablas de contingencia con el estadístico Chi-Cuadrado de Pearson, V de Cramer y el análisis de los residuos tipificados corregidos para la comprobación de las hipótesis.

\section{Resultados}

\section{Actitudes hacia la inclusión de estudiantes discapacitados en la Universidad}

La $\mathrm{H} 1$ planteaba que los estudiantes presentarían actitudes favorables hacia la inclusión de personas con discapacidad en la universidad.

El primer bloque del cuestionario perseguía examinar las actitudes de los estudiantes sobre la presencia de compañeros con discapacidad en las aulas. Para ello, se solicitó a los encuestados que indicaran su grado de acuerdo (1, Muy en desacuerdo; 5 , Totalmente de acuerdo) con un conjunto de afirmaciones.

El 70,8\% de los alumnos estaba totalmente de acuerdo con que "Se debe facilitar el ingreso a la universidad a los estudiantes con discapacidad" $(\mathrm{M}=4,53$. $\mathrm{Dt}=0,89)$. Apenas un $5 \%$ de los encuestados manifestó algún grado de desacuerdo con esta cuestión.

De nuevo hallamos alrededor de un 5\% de los alumnos con una opinión desfavorable a la inclusión de estudiantes con discapacidad en la universidad, pues indicaron algún grado de acuerdo con que "La Universidad no es el lugar más adecuado para la formación académica y profesional de los estudiantes con discapacidad" $(\mathrm{M}=1,44 . \mathrm{Dt}=0,84)$. Por el contrario, el $72,1 \%$ de los alumnos expresaron su completo desacuerdo con la afirmación contenida en el ítem.

Sin embargo, cuando se preguntó si "La presencia de estudiantes con discapacidad en las aulas dificulta o ralentiza el rendimiento académico de los demás" $(\mathrm{M}=1,68$. $\mathrm{Dt}=1$ ), hubo mayor diversidad de opiniones a tenor de la desviación típica. Algo más de un $7 \%$ de los encuestados mostró algún grado de acuerdo con esta afirmación. Solo un $59,9 \%$ indicó su completo desacuerdo con que los estudiantes con discapacidad sean un lastre para el aprendizaje del resto del grupo.

La mayoría de los alumnos $(51,4 \%)$ expresó su total acuerdo con que "La presencia de estudiantes con discapacidad enriquece la convivencia dentro del aula" ( $M=4,24$. $\mathrm{Dt}=0,921)$, aunque de nuevo hubo mayor disparidad de opiniones. A pesar de eso, tan solo un porcentaje marginal, inferior al 3\%, declaró algún grado de desacuerdo con el ítem.

La RQ1 se preguntaba por la opinión de los estudiantes ante la posibilidad de trabajar en un grupo con estudiantes con discapacidad, así como sobre su percepción sobre el rendimiento académico de estos compañeros. 
Las actitudes son algo menos favorables cuando se estrecha el círculo sobre las relaciones interpersonales en el ámbito académico. Así lo demuestran las opiniones de los estudiantes sobre "Compartir grupo en trabajos de clase con alumnos con discapacidad". Aunque más de dos tercios de los encuestados no tendrían inconveniente en compartir trabajo de clase con sus compañeros y un porcentaje notable incluso presenta una disposición positiva, hallamos de nuevo un reducto de alumnos que "preferiría no compartir con ellos tareas académicas" (tabla 2).

\begin{tabular}{lc}
\hline Opinión sobre compartir tareas & Porcentaje \\
\hline $\begin{array}{l}\text { "Me gustaría compartir con ellos } \\
\text { tareas académicas" }\end{array}$ & $23,2 \%$ \\
\hline "Me da igual" & $69,2 \%$ \\
\hline $\begin{array}{l}\text { "Preferiría no compartir con ellos } \\
\text { tareas académicas" }\end{array}$ & $4,2 \%$ \\
\hline Total & $100,0 \%$ \\
\hline
\end{tabular}

Tabla 2. Opinión sobre compartir tareas académicas con estudiantes con discapacidad

Observamos también la opinión de los estudiantes sobre el "Rendimiento académico de los alumnos con discapacidad". El 70,8\% de los estudiantes manifestó que el éxito en los estudios de sus compañeros con discapacidad es "Como el de cualquier otra persona". Llama aquí la atención que el 18,5\% dijera que "No sabe" y que un $4,2 \%$ no quisiera responder la pregunta. Posiblemente, el sesgo de deseabilidad social jugó un papel relevante en este ítem. En cualquier caso, solo el 1,6\% de los alumnos expresó que "La mayoría de los estudiantes con discapacidad tiene un bajo rendimiento académico", frente al 4\% que indicó lo contrario.

\section{Las relaciones interpersonales en el aula}

La RQ2 preguntaba cuál era el grado de experiencia de los estudiantes en cuanto a relaciones en el aula con compañeros con discapacidad, así como por la calidad de dichas relaciones.

Las opiniones y actitudes no siempre se sustentan en el conocimiento de primera mano, en la experiencia directa con la realidad. Por ello, preguntamos a los estudiantes si habían cursado alguna vez una asignatura junto a compañeros que presentaran alguna discapacidad. La respuesta fue afirmativa para el $47,4 \%$ de los alumnos de la muestra $(n=294)$. Interpelamos exclusivamente a este grupo sobre la "Calidad de las relaciones con sus compañeros con discapacidad en el día a día". Más del $81 \%$ de los encuestados la calificó positivamente y solo algo menos del 3\% reconoció que su relación en el día a día era regular. Sin embargo, el 15,6\% afirmó que no tenía relaciones con estos compañeros.

Asimismo, les preguntamos acerca de su "Posición a la hora de entablar relaciones personales con sus compañeros con discapacidad". El 71,3\% de los encuestados que alguna vez había coincidido en una asignatura con estudiantes que presentaban alguna discapacidad dijo que trata a esos compañeros como a los demás. El resto indicó que 
adopta una postura diferente dependiendo del tipo de discapacidad que presenta el compañero. Las demás opciones de respuesta apenas fueron secundadas.

\section{Conocimiento sobre la discapacidad}

La RQ3 indagaba acerca del conocimiento sobre la discapacidad y las vías de acceso al mismo por parte de los estudiantes.

En la medida en que quizás un mayor grado de conocimiento e información sobre la discapacidad pudiera estar relacionado con el desarrollo de actitudes favorables, preguntamos también a los encuestados sobre su "Percepción acerca del grado de información respecto a la discapacidad". Dos tercios de los alumnos se sienten solo algo o poco informados (tabla 3 ) mientras que el $10,7 \%$ se considera nada informado sobre el tema. Evaluamos el grado de conocimiento de los estudiantes sobre tres servicios de la universidad dirigidos al colectivo con discapacidad. Los resultados indicaron que solo el 20\% conocía la existencia del Servicio de Apoyo al Alumnado con Discapacidad, un porcentaje idéntico conocía la figura del alumno colaborador y un 38\% tenía constancia de la existencia de la Guía de Accesibilidad de la Universidad de Málaga.

\begin{tabular}{lc}
\hline Grado de información & Porcentaje \\
\hline Nada informado & $10,7 \%$ \\
\hline Poco informado & $33,8 \%$ \\
\hline Algo informado & $34,6 \%$ \\
\hline Bastante informado & $15,4 \%$ \\
\hline Muy informado & $3,2 \%$ \\
\hline No sé & $2,2 \%$ \\
\hline Total & $100,0 \%$ \\
\hline
\end{tabular}

Tabla 3. Percepción acerca de su grado de información respecto a la discapacidad

La mayoría del colectivo estudiantil $(76,7 \%)$ respondió afirmativamente a la pregunta sobre si le gustaría recibir información sobre la discapacidad. Lamentablemente, tan solo el $20 \%$ de los alumnos manifestó haber recibido en alguna ocasión cualquier tipo de comunicación sobre esta cuestión por parte de la Universidad. Interpelamos exclusivamente a estos estudiantes $(n=117)$ sobre el tipo de información que les había sido facilitada por la institución. Los resultados señalaron que un tercio de los alumnos habían cursado alguna asignatura en cuyo temario se abordaban aspectos relacionados con la discapacidad; un 31,8\% también accedió a publicaciones que trataban de alguna manera el asunto y un $20,4 \%$ asistió a una jornada, seminario o mesa redonda en la que se habló sobre discapacidad. Otro tipo de actividades como cursos de formación o visitas guiadas a centros o instituciones relacionadas con la discapacidad obtuvieron mucha menos penetración entre el alumnado: ambas fueron señaladas por el $7 \%$ de los encuestados. 


\section{Asociación entre variables}

La $\mathrm{H} 2$ planteaba la existencia de una asociación positiva entre las actitudes favorables hacia el colectivo de estudiantes con discapacidad y el grado de información recibido sobre discapacidad.

Hallamos correlación entre el grado de información recibido sobre discapacidad y el acuerdo con que "La presencia de estudiantes con discapacidad enriquece la convivencia dentro del aula" $(r=0,110 . p=0,009)$ a un nivel de significación de 0,01. Del mismo modo, el grado de información estaba correlacionado con el acuerdo sobre que "Se debe facilitar el ingreso a la universidad a los estudiantes con discapacidad" $(\mathrm{r}=0,083 \cdot \mathrm{p}=0,047)$ a un nivel de significación de 0,05 . Además, encontramos una correlación inversa con el acuerdo respecto a que "La presencia de estudiantes con discapacidad en las aulas dificulta o ralentiza el rendimiento académico de los demás" $(r=-0,123 . p=0,003)$ a un nivel de significación de 0,01 ; esto es, cuanto mayor era el nivel de información recibido por el estudiante, mayor desacuerdo mostraba con que los alumnos con discapacidad pudieran ser un lastre para el resto.

Por otra parte, se detectó una asociación entre el grado de información ${ }^{4}$ sobre discapacidad y la "Opinión sobre compartir grupo en trabajos de clase" [ $\chi^{2}$ (4, $\mathrm{N}=572)=14,36 . \mathrm{p}=0,006, \mathrm{~V}=0,112]$, indicando que cuanto mayor es el grado de información de los estudiantes, más favorables son sus actitudes hacia los compañeros con discapacidad. De acuerdo con los residuos tipificados corregidos, el porcentaje de estudiantes con alto grado de información a los que les gustaría compartir tareas académicas con alumnos discapacitados fue significativamente superior al de estudiantes con bajo grado de información y viceversa (ver tabla 4).

\begin{tabular}{|c|c|c|c|}
\hline $\begin{array}{l}\text { Porcentajes de columna. Las celdas sombreadas } \\
\text { señalan los valores significativos. }\end{array}$ & $\begin{array}{c}\text { Más bien } \\
\text { poco } \\
\text { informado }\end{array}$ & $\begin{array}{c}\text { Algo } \\
\text { informado }\end{array}$ & $\begin{array}{c}\text { Más bien } \\
\text { bastante } \\
\text { informado }\end{array}$ \\
\hline $\begin{array}{l}\text { "Me gustaría compartir con ellos tareas } \\
\text { académicas" }\end{array}$ & $17,8 \%$ & $27,1 \%$ & $34,5 \%$ \\
\hline "Me da igual" & $76,8 \%$ & $70,0 \%$ & $60,9 \%$ \\
\hline $\begin{array}{l}\text { "Prefiero no compartir con ellos tareas } \\
\text { académicas" }\end{array}$ & $5,4 \%$ & $3,0 \%$ & $4,5 \%$ \\
\hline Total & $100,0 \%$ & $100,0 \%$ & $100,0 \%$ \\
\hline
\end{tabular}

Tabla 4. Diferencias en la opinión sobre compartir tareas según el grado de información

Asimismo, detectamos una asociación entre el grado de información recibido y la "Opinión sobre el rendimiento académico de los estudiantes con discapacidad" [ $\chi 2$ (6,

\footnotetext{
${ }^{4}$ La variable "Grado de información recibida sobre discapacidad" originariamente constaba de cinco categorías. Sin embargo, fue recodificada en una variable de tres categorías (1, Más bien poco informado; 2, Algo informado; 3, Más bien bastante informado) para poder cumplir el requisito de la prueba de Chi Cuadrado que exige que menos del $25 \%$ de las casillas presenten una frecuencia esperada inferior a cinco.
} 
$\mathrm{N}=572)=21,74 . \mathrm{p}=0,001, \mathrm{~V}=0,137]$. A tenor de los residuos tipificados corregidos, el porcentaje de estudiantes mejor informados que atribuía un alto rendimiento académico a sus compañeros discapacitados fue significativamente superior al de los alumnos con un grado de información más bajo (ver tabla 5). Del mismo modo, aquellos peor informados tendían a pensar en menor medida que el resto de estudiantes que sus compañeros con discapacidad rinden como cualquiera, dependiendo de los casos.

\begin{tabular}{|c|c|c|c|}
\hline $\begin{array}{l}\text { Porcentajes de columna. Las celdas sombreadas } \\
\text { señalan los valores significativos. }\end{array}$ & $\begin{array}{c}\text { Más bien } \\
\text { poco } \\
\text { informado }\end{array}$ & $\begin{array}{c}\text { Algo } \\
\text { informado }\end{array}$ & $\begin{array}{c}\text { Más bien } \\
\text { bastante } \\
\text { informado }\end{array}$ \\
\hline "La mayoría tiene un alto rendimiento" & $3,1 \%$ & $5,4 \%$ & $10,0 \%$ \\
\hline "La mayoría tiene un bajo rendimiento" & $1,5 \%$ & $1,5 \%$ & $0,9 \%$ \\
\hline "Como cualquier persona, depende" & $69,8 \%$ & $77,5 \%$ & $80,0 \%$ \\
\hline No sé & $25,6 \%$ & $15,7 \%$ & $9,1 \%$ \\
\hline Total & $100,0 \%$ & $100,0 \%$ & $100,0 \%$ \\
\hline
\end{tabular}

Tabla 5. Diferencias en la opinión sobre el rendimiento académico según grado de información

A la vista de los datos, los resultados apoyan la H2. Por tanto, podemos afirmar que cuanto mayor es el grado de información de los estudiantes, mejores son sus actitudes hacia sus compañeros con discapacidad.

La H3 establecía que los estudiantes que tienen un conocido con discapacidad en su entorno cercano presentarían actitudes más favorables hacia el trabajo colaborativo y el rendimiento académico de alumnos con discapacidad.

Hallamos evidencias de asociación entre las variables. Los resultados indicaron que la opinión sobre compartir grupo en trabajos de clase con estudiantes con discapacidad variaba significativamente entre los estudiantes que conocían a alguna persona con discapacidad de su entorno y los que no $\left[\chi^{2}(2, \mathrm{~N}=569)=9,131\right.$. $\left.\mathrm{p}=0,010, \mathrm{~V}=0,127\right]$. Los residuos tipificados corregidos señalaron que el porcentaje de alumnos a quienes gustaría compartir grupo con un compañero con discapacidad era significativamente más alto entre quienes tenían un conocido que entre los que no mantenían ninguna relación con una persona con discapacidad (ver tabla 6).

\begin{tabular}{|c|c|c|}
\hline $\begin{array}{l}\text { Porcentajes de columna. } \\
\text { Las celdas sombreadas señalan los valores } \\
\text { significativos. }\end{array}$ & $\begin{array}{l}\text { Conoce a } \\
\text { alguien }\end{array}$ & $\begin{array}{l}\text { No tiene } \\
\text { conocido }\end{array}$ \\
\hline $\begin{array}{l}\text { "Me gustaría compartir con ellos tareas } \\
\text { académicas" }\end{array}$ & $29 \%$ & $18,3 \%$ \\
\hline "Me da igual" & $66,4 \%$ & $77,5 \%$ \\
\hline $\begin{array}{l}\text { "Preferiría no compartir con ellos tareas } \\
\text { académicas" }\end{array}$ & $4,6 \%$ & $4,2 \%$ \\
\hline Total & $100,0 \%$ & $100,0 \%$ \\
\hline
\end{tabular}

Tabla 6. Diferencias en la opinión sobre compartir tareas según la experiencia previa en el trato con personas con discapacidad 
Por otra parte, encontramos también una asociación significativa entre la experiencia directa y la opinión sobre el rendimiento académico de los estudiantes con discapacidad $[\chi 2(3, N=573)=12,86 . p=0,005, V=0,150]$. A la luz de los residuos tipificados corregidos, el porcentaje de estudiantes con un conocido con discapacidad que opinaba que el rendimiento de los alumnos con discapacidad es "como el de cualquier otra persona" era significativamente mayor que el de los estudiantes que no tenían a ningún conocido de ese colectivo (ver tabla 7).

\begin{tabular}{lcc}
\hline $\begin{array}{l}\text { Porcentajes de columna. } \\
\text { Las celdas sombreadas señalan los } \\
\text { valores significativos. }\end{array}$ & $\begin{array}{c}\text { Conoce a } \\
\text { alguien }\end{array}$ & $\begin{array}{l}\text { No tiene } \\
\text { conocido }\end{array}$ \\
\hline "La mayoría tiene un alto rendimiento" & $6,1 \%$ & $3,4 \%$ \\
\hline "La mayoría tiene un bajo rendimiento" & $1,6 \%$ & $1,9 \%$ \\
\hline "Como cualquiera, depende" & $78,3 \%$ & $69,7 \%$ \\
\hline "No sé" & $13,9 \%$ & $25,0 \%$ \\
\hline & $100,0 \%$ & $100,0 \%$ \\
\hline
\end{tabular}

Tabla7. Diferencias en la opinión sobre el rendimiento académico según la experiencia previa en el trato con personas con discapacidad

De acuerdo a los datos, los resultados arrojados por los análisis apoyan la H3. Por tanto, podemos afirmar que los estudiantes con experiencia previa, esto es, que conocen a personas con discapacidad, son más proclives a trabajar colaborativamente con compañeros de dicho colectivo, valorándolos además con mayor justicia.

\section{Discusión y conclusiones}

La universidad se dibuja como un espacio de fomento de la educación superior inclusiva, promotora de la igualdad de oportunidades y las actitudes positivas, integradoras y de convivencia con la discapacidad. Año tras año el número de estudiantes con algún tipo de discapacidad crece en las universidades españolas, y se hacen necesarios nuevos estudios que midan el grado de normalización que alcanza su presencia. Los resultados obtenidos en la presente investigación permiten concluir que los universitarios malagueños presentan actitudes favorables al ingreso de estudiantes con discapacidad en la Universidad; sólo el 5\% muestra algún grado de desacuerdo con que se deba facilitar su ingreso, mientras que un residual $1 \%$ prefiere que no haya compañeros en el aula. Especialmente significativa resulta la relación que existe entre un mayor grado de información y conocimiento y una actitud más inclusiva. En este sentido, y coincidiendo con estudios previos (Alonso, Navarro y Lidón, 2008; Suriá, 2011; Suriá, Bueno y Rosser, 2011), se ha encontrado que los alumnos que, en su entorno más próximo, conocen a personas que tienen una discapacidad muestran una actitud más positiva ante ellas que las que no las conocen. Se confirma así que la convivencia es un factor determinante para mejorar las actitudes (Novo y Muñoz, 2012). 
Respecto a la opinión sobre los posibles efectos que tiene la inclusión de estudiantes con discapacidad en el rendimiento académico del resto, en torno al $7 \%$ de los encuestados mostró algún grado de acuerdo con que ralentizan o dificultan el trabajo de clase, mientras que el $60 \%$ mostró su completo desacuerdo. Fue uno de los ítems que suscitó mayor controversia. Por otra parte, los resultados sobre la percepción de su rendimiento académico son afines a los de la mayoría de estudios comparativos entre estudiantes con y sin discapacidad, que evidencian que no existen diferencias tan significativas entre ambos grupos. Así Jelfs y Richardson (2010) concluyen que el impacto de la discapacidad en la percepción de la calidad académica parece ser muy leve (en Zubillaga del Río y Alba, 2013).

A la luz de estos hallazgos, y a pesar de que hayan sido positivos, parece razonable que las universidades españolas refuercen las líneas de trabajo para reducir el porcentaje de estudiantes que presentan actitudes desfavorables o poco inclusivas (en nuestro estudio en torno el 5 y el 7\%, según el aspecto concreto). Por un lado, sería interesante ofrecer alguna asignatura en los planes de estudio que trate sobre el tema, o que éste sea un eje transversal en el currículo. No obstante, puesto que es difícil ajustar esta materia a los programas formativos de determinadas áreas de conocimiento, se plantea otra línea de acción: campañas de divulgación y sensibilización entre la comunidad universitaria. Por ejemplo, sería pertinente incluir a profesionales que presenten alguna discapacidad entre los conferenciantes habituales de los centros. Así, se cultivarían actitudes favorables sobre las capacidades y rendimiento académico de los estudiantes con discapacidad.

Asimismo, las universidades deberían promover el contacto directo de su comunidad universitaria con personas con discapacidad. Los programas de alumnos colaboradores ${ }^{5}$ son una buena idea, pero generalmente solo movilizan a personas que ya están sensibilizadas. Quizás el planteamiento deba invertirse en algunos casos para pasar de la labor asistencial al empoderamiento. Algunos estudiantes con discapacidad de los últimos cursos pueden actuar como cicerones o tutores de otros recién llegados. Con esta medida se fomentaría el empoderamiento del colectivo con discapacidad al mismo tiempo que se contribuiría a moldear las percepciones y actitudes de los estudiantes del primer curso sobre las capacidades de sus compañeros. Del mismo modo, sería interesante que los estudiantes con discapacidad se implicaran en mayor medida, por ejemplo, en la representación estudiantil, formando parte de las asociaciones y demás grupos que participan en la política universitaria, visibilizando la integración y los valores del colectivo. Son tan sólo algunas de las acciones para atender a la diversidad con pertinencia y acción estratégica de calidad, necesarias para erradicar las barreras simbólicas e interpretativas-culturales presentes en cada institución de educación superior, sobre las que reflexiona Ocampo (2011) y que constituyen un punto crítico para la puesta en marcha de estrategias que ejercitan activamente el derecho a la educación de este colectivo de estudiantes.

\footnotetext{
${ }^{5}$ En estos programas, estudiantes sin discapacidad colaboran como voluntarios en la asistencia a alumnos con discapacidad. Ayudan en tareas como el desplazamiento dentro del campus, la toma de apuntes y notas, etcétera.
} 
Por último, respecto al alcance de la investigación, hay que recordar que el tamaño de la muestra es notable; no obstante, los resultados no pueden extrapolarse al conjunto de los estudiantes universitarios españoles. Sin embargo, suponen: por un lado, una nueva evidencia empírica que aporta validez a estudios realizados en otras poblaciones universitarias; por otro, un punto de partida de gran utilidad para el desarrollo posterior de la investigación y del proyecto de innovación docente en el que se inscribe. En el futuro, sería interesante examinar distintas variables en las actitudes de los estudiantes universitarios, como pueden ser el tipo de discapacidad, así como el grado en el que se manifiesta, ya que éstas podrían influir en las opiniones y conductas (como ya hicieron en 1983 Stovall y Sedlacek). Incluso ir más allá y tener en cuenta otras variables que puedan influir en la percepción de la diversidad como: el género, la procedencia del alumnado, sus creencias religiosas, el nivel socioeconómico, etcétera. Asimismo, test conductuales para recabar información sobre la actuación de los sujetos ante los estudiantes con discapacidad. Por otra parte, para los próximos acercamientos al objeto de estudio, es preciso implementar instrumentos más complejos para la medición de las variables con objeto de mejorar la fiabilidad y validez, así como coordinar los esfuerzos de investigadores de varias universidades. De esta forma, los resultados obtenidos cobrarían mayor significación en un análisis comparativo que incluyese a un número mayor de instituciones españolas.

\section{Referencias bibliográficas}

AJZEN, I. (1991). The theory of planned behavior. Organizational Behavior and human Decision Processes, 50, 179-211.

ALONSO, M.J., NAVARRO, R. Y LIDÓN, V. (2008). Actitudes hacia la diversidad de estudiantes universitarios. Comunicación presentada en las XIII Jornadas de Fomento de la Investigación, Universitat Jaume I, Castellón, España.

ANDREU, A., PEREIRA, A. y RODRÍGUEZ, V.M. (2010). Guía de adaptaciones y recursos para estudiantes con discapacidad. Madrid: UNED.

ANTHONY, W. A. (1972). Societal rehabilitation: Changing society's attitudes toward the physically and mentally disabled. Rehabilitation Psychology, 19, 3, 117-126.

BORLAND, J. y JAMES, S. (1999). The learning experience of students with disabilities in higher education. A case study of a UK university. Disability \& Society, 14 (1), 85-101.

BUENO, A. y MIRAMBEL, E. (2003). Escala de actitudes hacia la discapacidad en la universidad: Presentación. Encuentros en Psicología Social, 1, 36-39.

CENTER, Y. Y WARD, J. (1987). Teachers' attitutes towards the integration of disabled children into regular schools. Exceptional Child, 34, 41-56. doi: $10.1080 / 0156655870340105$.

CERMI (2014). Medidas de acción positiva para el acceso y apoyo al personal docente e investigador con discapacidad de la Universidad de... Modelo de 
reglamento universitario. Comité Español de Representantes de Personas con Discapacidad.

CLORE, G.L. Y JEFFERY, K. M. (1972). Emotional role playing, attitude change, and attraction toward a disabled person. Journal of Personality and Social Psychology, $23,1,105-111$.

EVANS, H. (1976). Changing attitudes toward disabled persons: An experimental study. Rehabilitation Counseling Bulletin, 19, 4, 572-579.

FUNDACIÓN UNIVERSIA (2014). Guía de Atención a la Discapacidad en la Universidad. Fundación Universia.

FRITH, G. Y MITCHELL, J. (1981). The attitudes of non handicapped students toward mildly retarded: A consideration in placement decisions. Education and Training of the Mentally Retarded, 16, 79-83.

GITLOW, L. (2001). Occupational therapy faculty attitudes toward the inclusión of students with disabilities in their educational programs. The Occupational Therapy Journal of Research, 21, 115- 131.

JELFS, A. Y RICHARDSON, J.T. (2010). Perceptions of Academic Quality and Approaches to Studying among Disabled and Non disabled Students in Distance Education. Studies in Higher Education, 35, 5, 593-607. doi: $10.1080 / 03075070903222666$.

LUQUE, D. Y RODRÍGUEZ, G. (2007). Guía de orientación al profesorado del alumnado con discapacidad en la Universidad de Málaga. Málaga: Universidad de Málaga.

LUQUE DE LA ROSA, A. Y GUTIÉRREZ CÁCERES, R. (2014). La integración educativa y social del alumnado con discapacidad en el EEES: Universidad de Bolonia. Revista Complutense de Educación, 25, 1, 153-175. http://dx.doi.org/10.5209/rev_RCED.2014.v25.n1.41344

MARTÍNEZ LIÉBANA, I., STORCH, J., SÁNCHEZ, P. Y DELGADO, C. (2004). Pautas básicas para facilitar la integración de las personas con discapacidad. Madrid: Universidad Complutense de Madrid.

MORIÑA DIEZ, A. et al. (2013). El profesorado en la universidad ante el alumnado con discapacidad: ¿Tendiendo puentes o levantando muros? Revista de Docencia Universitaria, 11 (3), 423-442.

MOSWELA, E. Y MUKHOPADHYAY, S. (2011). Asking for too much? The voices of students with disabilities in Botswana. Disability \& Society, 26 (3), 307-319.

NOVO, I., MUÑOZ, J.M. y Calvo, C. (2011). Análisis de las actitudes de los jóvenes universitarios hacia la discapacidad: Un enfoque desde la Teoría de la Acción Razonada. Relieve, $17, \quad 2 . \quad$ Recuperado de http://www.uv.es/RELIEVE/v17n2/RELIEVEv17n2_5.htm 
NOVO, I. Y LÓPEZ, M.D. (2013). La búsqueda de trabajo normalizado para las personas con discapacidad: un análisis del papel de los compañeros como factor de inclusión social. Comunicación presentada en el IV Congreso de la Red Española de Política Social, Universidad de Alcalá de Henares, Madrid, España.

NOVO, I. Y MUÑOZ, J.M. (2012). Los estudiantes universitarios ante la inclusión de sus compañeros con discapacidad. Revista Española de Orientación y Psicopedagogía, 23, 2, 105-122. Recuperado de http://redined.mecd.gob.es/xmlui/bitstream/handle/11162/95047/00820123017438. pdf? sequence $=1$

OCAMPO, A. (2011). Inclusión de estudiantes en situación de discapacidad a la educación superior. Desafíos y oportunidades. Revista Latinoamericana de Educación Inclusiva, 6 (2), 227-239.

POLO, M.T. Y LÓPEZ, M.D. (2006). Actitudes hacia las personas con discapacidad de estudiantes de la Universidad de Granada. Revista Española de Orientación y Psicopedagogía, 17, 2, 195-211. Recuperado de http://www.uned.es/reop/pdfs/2006/17-2-2\%20-\%20Maria\%20Tamara\%20Polo.pdf

REINDAL, S. M. (1995). Some problems encountered by disabled students at the University of Oslo - whose responsibility? The European Journal of Special Needs, 10 (3), 227-241.

SÁNCHEZ PALOMINO, A. (Coord.) (2009). Integración educativa y social de los estudiantes con discapacidad en la Universidad de Almería. Almería: Universidad de Almería.

- (2011). La Universidad de Almería ante la integración educativa y social de los estudiantes con discapacidad: Ideas y actitudes del personal docente e investigador. Revista de Educación, 354, 575-603. Recuperado de http://www.revistaeducacion.educacion.es/re354/re354_23.pdf

SANTOS, M.A. Y LORENZO, M.M. (2010). La dimensión cívica en el desarrollo formativo de los estudiantes universitarios. Revista Electrónica de Investigación Educativa. Recuperado http://redie.uabc.mx/index.php/redie/article/view/250/411

SERVICIO CENTRAL DE INFORMÁTICA (2010). Estadísticas de alumnos. Grado $1^{\circ}$ y $2^{\circ}$ ciclo. Curso 2009-2010. Málaga: Universidad de Málaga.

STOVALL, C. Y SEDLACEK, W.E. (1983). Attitudes of male and female university students toward students with different physical disabilities. Journal of College Student Personnel, 24, 4, 325-330.

SURIÁ, R. (2011). Comparative analysis of student's attitudes toward their classmates with disabilities. Electronic Journal of Research in Educational Psychology, 9, 197216.

-, BUENO, A. Y ROSSER, A. M. (2011). Prejuicios entre los estudiantes hacia las personas con discapacidad: Reflexiones a partir del caso de la Universidad de 
Alicante. Alternativas, 18, 75-90. Recuperado de http://193.145.233.67/dspace/bitstream/10045/18711/1/Alternativas_18_04.pdf

TINKLIN, T. Y HALL, J. (1999). Getting round obstacles: disabled students' experiences in higher education in Scortland. Studies in Higher Education, 24: 183194.

YUKER, H. E. Y BLOCK, J. R. (1986). Research with the Attitude Toward Disabled Persons scales (ATDP) 1960-1985. Hofstra University.

ZUBILLAGA DEL RÍO, A. Y ALBA, C. (2013). La discapacidad en la percepción de la tecnología entre estudiantes universitarios. Comunicar, 20, 40,165-172. doi: http://dx.doi.org/10.3916/C40-2013-03-07.

\section{Agradecimientos:}

Este trabajo contó con la colaboración inestimable de los estudiantes de la Universidad de Málaga: María García Sánchez, Tamara Garnica Muñoz, Javier Pérez Cuerva, Irene Pérez de Navarro Martín y Teresa Velasco Castillo, quienes participaron en el pretest del cuestionario y en el proceso de recogida de datos.

\section{Correspondencia con los autores}

Eugenia GONZÁLEZ CORTÉS

Facultad de Ciencias de la Comunicación.

Campus de Teatinos s/n.

29071. Málaga.

e-mail: eugenia@uma.es

Sergio ROSES CAMPOS

Facultad de Ciencias de la Comunicación.

Campus de Teatinos s/n. 29071.

Málaga.

e-mail: roses@uma.es 